\title{
To Study the Effectiveness of Massage Therapy Combined with Pharmacological Intervention for the Treatment of Headache in a Tertiary Care Centre at Shimoga, India
}

\author{
Bhuvana Chandrashekarappa Revappala ${ }^{1}$, Sridhar Mallanaik², Vibha Konthanur Vijaykumar33, \\ Suresh Krishnappa Kudlumallige ${ }^{4}$, Sarala Nedige Eshwarappa ${ }^{5}$ \\ 1, 2, 3, 4,5 Department of Psychiatry, Shimoga Institute of Medical Sciences, Shimoga, Karnataka, India.
}

\section{ABSTRACT}

\section{BACKGROUND}

Headache is the most common disabling condition worldwide that can be treated with massage therapy. But due to not practising by us there is a gap in applying massage therapy, as a non-pharmacological, along with pharmacological intervention for the treatment of headache which improves symptoms. There is negligible information on the effectiveness of that combined therapy to treat headaches. We wanted to study the effectiveness of combined therapy in a study group as compared to the control group and also to check the statistical significance among the study group (combined therapy) and control group (mono-therapy).

\section{METHODS}

A total of 30 study groups and 30 control groups of both sex with the age group of 18 - 65 years were included. The study group was explained about the structured massage therapy and encouraged to take the pharmacological drug along with it. The severity of headache was determined by using the HIT- 6 scale and the subjects were assessed by themselves using the measure yourself medical outcome profile (MYMOP) questionnaire. The study was conducted in tertiary care hospitals which included both in-patient and out-patient departments. It was a prospective study. Descriptive and Inferential statistics were used.

\section{RESULTS}

Compared with the initial visit, the severity of headache symptoms significantly reduced within two weeks of the massage protocol along with pharmacological intervention as compared to the control group. The severity of associated symptoms also reduced significantly following massage therapy.

\section{CONCLUSIONS}

Our study proved that combined therapy was very effective as compared to the control group who were on monotherapy. Needs to apply it clinically in future. Since both act at different sites, our study proved that combined therapy is effective in reducing headache along with associated symptoms significantly.

\section{KEY WORDS}

Massage Therapy, Headache, Amitriptyline, MTrPs (Myofascial Trigger Points)
Corresponding Author: Dr. Sridhar Mallanaik, \#605, Sangam Doctors Quarters, Near IMA House, SIMS Campus, Sagar Road, Shimoga - 577201, Karnataka, India.

E-mail:drsridhar2@gmail.com

DOI: $10.14260 /$ jemds/2021/633

How to Cite This Article:

Revappala BC, Mallanaik S, Vijaykumar $V K$, et al. To study the effectiveness of massage therapy combined with pharmacological intervention for the treatment of headache in a tertiary care centre at Shimoga, India. J Evolution Med Dent Sci 2021;10(36):3103-3108, DOI: 10.14260/jemds/2021/633

Submission 18-02-2021, Peer Review 16-07-2021, Acceptance 23-07-2021, Published 06-09-2021.

Copyright (C) 2021 Bhuvana Chandrashekappa Revappala et al. This is an open access article distributed under Creative Commons Attribution License [Attribution 4.0 International (CC BY 4.0)] 


\section{BACKGROUND}

Headache is the most common neurological and disabling condition worldwide which is usually associated with comorbid psychiatric disorders that worsen the pre-existing headache or vice-versa. ${ }^{1}$ Although treatments are available for treating headaches, massage therapy has been used effectively as a therapeutic regimen for relieving pains like headache for many centuries. ${ }^{2}$ But it is not being practised clinically. Massage therapy is benefited by improving blood supply to muscles, allowing toxins to be removed; and reduced anxiety and depression which leads to a sense of "well-being"- by releasing endorphins, the brain chemicals (Neurotransmitters). ${ }^{3}$ Hence massage is mood-lifting. Massage is a great way to let your stress disappear. Massage works by relieving tight, strained muscle fibres which are most predominant in myofascial trigger points (MTrPs). These myofascial trigger points are the hyperirritable spots in skeletal muscles that are hypersensitive palpable nodules in a taut band. During headache, there is a sustained muscle contraction in MTrPs, which leads to hypoxia and ischemia of muscles that causes headache by stimulating pain receptors. $4,5,6$

Headache can be categorized based on the underlying health condition that is responsible for pain. It is primary or secondary as per the International Headache Society notes. ${ }^{7}$ Frequent or severe headaches can affect a person's quality of life. ${ }^{8}$ The pain receptors for headaches are most predominant in head and neck muscles, extra-cranial arteries and veins, cranial and spinal nerves, meninges, falx cerebri, eyes and ears, which send signals to the brain and hence sense pain, whereas the brain itself lack pain receptors. These pain receptors are often stimulated by blood vessel spasms, dilated blood vessels, inflammation or infection of meninges and muscular tension which send signals to the brain.9,10,11

The intensity of pain will vary according to the factors like location (attachment points are more sensitive, degree of trigger points irritability, active or latent trigger points, sites of trigger points i.e.; some areas are more sensitive. Other factors are associated with brain damage, host tissue stiffness, ageing chronicity of trigger points. ${ }^{12}$

Two main types of trigger points are described, active trigger points are those that may be responsible for the presenting pain complaints. They may also be associated with less readily definable symptoms such as weakness, paraesthesia, or temperature change and they may have associated referred pains. Latent trigger points present with muscle shortening and pain occurs only on the application of external pressure. These trigger points may become activated by a variety of stimuli including poor posture, overuse or muscle imbalance. ${ }^{13,14,15}$

Christopher Quinn study has proved that massage therapy is effective in reducing the number of headaches per week in chronic tension headache sufferers. They said that the active MTrPs are located in the upper trapezius, sternocleidomastoid and temporalis muscles among chronic tension-type headaches (CTTH). Other muscles involved are pterygoids lateralis, medialis, scalenus anterior, medial and posterior, orbicularis oculi. This trigger points manifest as a headache. Physical findings of trigger points are small nodules of the size of pin head, pea-sized nodules, large lumps, several large lumps next to each other.
Headache can lead to days missed from work, reduced performance at work, use of healthcare services, use of pain medications, reduced levels of social activity, reduced levels of physical activity, and reduced performance of family responsibilities. ${ }^{16}$

With respect to the above explanation, we can conclude that both act at different sites i.e., massage therapy acts well at trigger points whereas drug (Amitriptyline) at different receptors levels, hence combined therapy is effective. But the study is negligible.

We wanted to study the effectiveness of combined therapy in a study group as compared to the control group and also check the statistical significance among the study group (combined therapy) and control group (monotherapy).

\section{METHODS}

It is a prospective study conducted from April 2020 to June 2020 in a tertiary care hospital of Shimoga which included both inpatient as well as outpatient departments.

\section{Sampling Method}

All the subjects of the study group and control group were selected by simple random, and were interviewed using the IHS classification system ${ }^{17}$ to diagnose the headache. They belonged to the age group of 18 - 65 years of both sexes. A total of 60 subjects met the criteria for headache and were divided into 30 study groups and 30 control groups. The study group received both massage regimens and $\mathrm{T}$. amitriptyline whereas the control group received $\mathrm{T}$. amitriptyline only. An organic brain lesion was excluded. Lack of availability during follow-up visits was also excluded and treatment in retrospective was ignored. Both groups were advised to come for follow-up after 2 weeks from the day they visited OPD / IPD. The study group subjects were encouraged to take $\mathrm{T}$. amitriptyline along with massage therapy. The procedure of massage had been advised to the caregiver by the massage therapist. Massage therapy consisted of circular movements of fingers over scalp, both clockwise and anticlockwise direction, pressing the trigger points located over scalp, neck and shoulder, as explained.

Trigger points are identified by stiffness in the affected muscle, spot tenderness (exquisite pain), a palpable taut nodule or bands, presence of referred pain, which may be hotter or colder than surrounding tissues. The skin over trigger points is often slightly warmer than surrounding skin due to increased metabolic automatic activity. Subjects who were taking massage were advised to take notes for each time of massage given by the caregiver and bring the recorded notes during follow-up visits. Since it was a simple technique the caregiver agreed to massage the subject twice a week for 2 weeks. Informed consent was taken.

\section{Massage Technique}

The patient was placed in a comfortable position, where the affected muscle could undergo full excursion. It consisted of manual palpation of the upper trapezius, 
sternocleidomastoid, sub-occipital, splenius capitis, levator scapulae, and temporalis muscles to locate and manually treat trigger points. When located, active trigger points were treated by pincer or flat palpation with just enough pressure to elicit referred pain or autonomic referral phenomena. That pressure was maintained on the trigger point until the client reported that the referral pain had dissipated or for a maximum of 2 minutes. Pressure on the active trigger point was then slowly eased to elicit vascular flushing. Sustained pressure was applied until the trigger points were felt soft. This could take seconds to minutes. The pressures on the trigger points were repeatedly increased until the next barriers were met and so on. This procedure was repeated 3 to 5 times on each trigger point. To achieve better results, one can try to change the direction of pressure during these repetitions. Typically, 6 active trigger points were treated in the time allotted. The most commonly employed clinical technique used to confirm the presence of an MTrP is manual palpation, although various diagnostic modules like several different imaging methods have been used to evaluate myofascial trigger points including microdialysis, magnetic resonance imaging, infrared thermography, electromyography and ultrasound. Of these options, ultrasound appeared to be the most promising diagnostic tool particularly if elastography by Doppler variance imaging was used. Due to its lack of availability, we used the manual palpation technique which remained as the best modality to assess MTrPs.

The effectiveness of the treatment was measured by using the HIT- 6 scale ${ }^{18}$ among both groups; both initial and followup visits have been applied by Psychiatrists. MYMOP is a scale, ${ }^{19}$ which can be administered by subjects themselves during initial and follow-up visits. The MYMOP questionnaire is an individualised or patient reported outcome measurement tool, that helps identify changes in their health status. It is of two types- initial form, during initial visits and MYMOP-2 during follow up visits. The subjects were asked to volunteer the most troublesome symptoms associated with their condition, a problem related to activity and their overall well-being on a seven-point numerical rating scale. It has been found that every subject showed improvements in all domains with respect to headaches. These improvements were statistically significant after two weeks of treatment. MYMOP questionnaires allowed the subjects to select up to two symptoms that were concerning them the most and subjectively assessed the change of these symptoms over time following a therapeutic intervention. Each item asked the patients to respond on a 7 - point scale $(0=$ as good as it could be, $6=$ as bad as it could be). The MYMOP2 questionnaire was shown to be moderately valid and highly reliable. ${ }^{19}$

The Headache Impact Test - 6 (HIT - 6) is used to measure the impact headaches have on your ability to function on the job and in a social situation. The HIT- 6 consists of six items: pain, social functioning, role functioning, vitality, cognitive functioning, and psychological distress. ${ }^{18}$ The patient answers each of the six related questions using one of the following five responses: "never", "rarely", "sometimes", "very often", or "always". The disability was quantified using the following four impact grades based on the obtained HIT- 6 score (1) little-to-no impact (HIT - 6 score: 36 - 49), moderate impact (HIT - 6 score: 50 - 55), substantial impact (HIT - 6 score: 56 -
59), and severe impact (HIT-6 score: 60 - 78). These responses are summed to produce a total HIT-6 score that ranges from 36 to 78, where a higher score indicates a greater impact of headache on the daily life of the respondent. Extensive testing has shown the HIT- 6 to be highly reliable and internally consistent, and it has been translated into several languages. ${ }^{18}$ Ethical clearance was obtained from the institutional ethics committee.

\section{Data Analysis}

Both descriptive and inferential statistics were used. Hypothesis test - (t-test and chi-square test) was considered.

\section{RESULTS}

Study results are tabulated as below

$\mathrm{H}_{\mathrm{o}}=$ Null Hypothesis states that there are equal effects on the study group and control group.

$\mathrm{H}_{\mathrm{a}} \neq$ Alternative Hypothesis states that there are no equal effects on the study group and control group.

Mean of the study group
(Before Treatment)
Grades from MYMOP Scale
454665445666546
644555654455666
Mean $=X_{n} / n=152 / 30=5.2$

Mean of the control group (Before Treatment) Grades from MYMOP Scale 465466564456654 456654465664564 Mean $=X_{n} / n=152 / 3$

Mean of the study group
(After-Treatment)
Grades from MYMOP Scale
010201102110010
101120011210001
Mean $=X_{n} / n=21 / 30=0.7$

Mean of the control group (After-Treatment)

Grades from MYMOP Scale 231033221322323 323223122330132

Mean $=X_{n} / n=64 / 30=2.1$

\begin{tabular}{|c|c|c|c|c|c|}
\hline \multicolumn{3}{|c|}{ After Treatment } & \multicolumn{3}{|c|}{ Before Treatment } \\
\hline $\mathbf{X}_{2}$ & $\mathbf{X}_{2}-\mathbf{X}_{\text {bar }}$ & $\left(X_{2}-X_{\text {bar }}\right)^{2}$ & $\mathbf{X}_{1}$ & $\mathbf{X}_{1}-\mathbf{X}_{\text {bar }}$ & $\left(X_{1}-X_{b a r}\right)^{2}$ \\
\hline 0 & $0-0.7=-0.7$ & 4.9 & 4 & $4-5.2=-1.2$ & 1.4 \\
\hline 1 & $1-0.7=+0.3$ & 9.0 & 5 & $5-5.2=-0.2$ & 0.04 \\
\hline 0 & $0-0.7=-0.7$ & 4.9 & 4 & $4.5 .2=-1.2$ & 1.4 \\
\hline 2 & $2-0.7=+1.3$ & 1.6 & 6 & $6-5.2=+0.8$ & 0.64 \\
\hline 0 & $0-0.7=-0.7$ & 4.9 & 6 & $6-5.2=+0.8$ & 0.64 \\
\hline 1 & $1-0.7=+0.3$ & 9.0 & 5 & $5-5.2=-0.2$ & 0.04 \\
\hline 1 & $1-0.7=+0.3$ & 9.0 & 4 & $4-5.2=-1.2$ & 1.4 \\
\hline 0 & $0-0.7=-0.7$ & 4.9 & 4 & $4-5.2=-1.2$ & 1.4 \\
\hline 2 & $2-0.7=+1.3$ & 1.6 & 5 & $5-5.2=-0.2$ & 0.04 \\
\hline 1 & $1-0.7=+0.3$ & 9.0 & 6 & $6-5.2=+0.8$ & 0.64 \\
\hline 1 & $1-0.7=+0.3$ & 9.0 & 6 & $6-5.2=+0.8$ & 0.64 \\
\hline 0 & $0-0.7=-0.7$ & 4.9 & 6 & $6-5.2=+0.8$ & 0.64 \\
\hline 0 & $0-0.7=-0.7$ & 4.9 & 5 & $5-5.2=-0.2$ & 0.04 \\
\hline 1 & $1-0.7=+0.3$ & 9.0 & 4 & $4-5.2=-1.2$ & 1.4 \\
\hline 0 & $0-0.7=-0.7$ & 4.9 & 6 & $6-5.2=+0.8$ & 0.64 \\
\hline 1 & $1-0.7=+0.3$ & 9.0 & 6 & $6-5.2=+0.8$ & 0.64 \\
\hline 0 & $0-0.7=-0.7$ & 4.9 & 4 & $4-5.2=-1.2$ & 1.4 \\
\hline 1 & $1-0.7=+0.3$ & 9.0 & 4 & $4-5.2=-1.2$ & 1.4 \\
\hline 1 & $1-0.7=+0.3$ & 9.0 & 5 & $5-5.2=-0.2$ & 0.04 \\
\hline 2 & $2-0.7=+1.3$ & 1.6 & 5 & $5-5.2=-0.2$ & 0.04 \\
\hline 0 & $0-0.7=-0.7$ & 4.9 & 5 & $5-5.2=-0.2$ & 0.04 \\
\hline 0 & $0-0.7=-0.7$ & 4.9 & 6 & $6-5.2=+0.8$ & 0.64 \\
\hline 1 & $1-0.7=+0.3$ & 9.0 & 5 & $5-5.2=-0.2$ & 0.04 \\
\hline 1 & $1-0.7=+0.3$ & 9.0 & 4 & $4-5.2=-1.2$ & 1.4 \\
\hline 2 & $2-0.7=+1.3$ & 1.6 & 4 & $4-5.2=-1.2$ & 1.4 \\
\hline 1 & $1-0.7=+0.3$ & 9.0 & 5 & $5-5.2=-0.2$ & 0.04 \\
\hline 0 & $0-0.7=-0.7$ & 4.9 & 5 & $5-5.2=-0.2$ & 0.04 \\
\hline 0 & $0-0.7=-0.7$ & 4.9 & 6 & $6-5.2=+0.8$ & 0.64 \\
\hline 0 & $0-0.7=-0.7$ & 4.9 & 6 & $6-5.2=+0.8$ & 0.64 \\
\hline 1 & $1-0.7=+0.3$ & 9.0 & 6 & $6-5.2=+0.8$ & 0.64 \\
\hline \multicolumn{6}{|c|}{ Table 1. Statistics of Study Group } \\
\hline
\end{tabular}

Total $=\sum\left(\mathrm{X}_{2}-\mathrm{X}_{\mathrm{bar}}\right)^{2}=187.1$, Total $=\sum\left(\mathrm{X}_{1}-\mathrm{X}_{\mathrm{bar}}\right)^{2}=19.20$

$\mathrm{S}_{2}=\sqrt{ }(187.1) / 30-1=\sqrt{187.1} / 29=2.5, \mathrm{~S}_{1}=\sqrt{19.20} / 29=0.66$ t-test ${ }_{1}=\mathrm{X}_{1}$ bar $-\mathrm{X}_{2 \mathrm{bar}} / \sqrt{\left(\mathrm{S}_{1}{ }^{2} / \mathrm{n}_{1}+\mathrm{S}_{2}{ }^{2} / \mathrm{n}_{2}\right)=21.08}$ 


\begin{tabular}{|c|c|c|c|c|c|}
\hline \multicolumn{3}{|c|}{ Before Treatment } & \multicolumn{3}{|c|}{ After Treatment } \\
\hline $\mathbf{X}_{1}$ & $\mathbf{X}_{\mathbf{1}}-\mathbf{X}_{\mathbf{b a r}}$ & $\left(X_{1}-X_{b a r}\right)^{2}$ & $\mathbf{X}_{2}$ & $\mathbf{X}_{2}-\mathbf{X}_{\text {bar }}$ & $\left(X_{2}-X_{\text {bar }}\right)^{2}$ \\
\hline 6 & $6-5.2=+0.8$ & 0.64 & 2 & $2-2.1=-0.1$ & 0.01 \\
\hline 6 & $6-5.2=+0.8$ & 0.64 & 3 & $3-2.1=+0.9$ & 0.81 \\
\hline 4 & $4-5.2=-1.2$ & 1.44 & 1 & $1-2.1=-1.1$ & 1.21 \\
\hline 5 & $5-5.2=-0.2$ & 0.04 & 0 & $0-2.1=-2.1$ & 4.41 \\
\hline 6 & $6-5.2=+0.8$ & 0.64 & 3 & $3-2.1=+0.9$ & 0.81 \\
\hline 4 & $4-5.2=-1.2$ & 1.44 & 3 & $3-2.1=+0.9$ & 0.81 \\
\hline 6 & $6-5.2=+0.8$ & 0.64 & 2 & $2-2.1=-0.1$ & 0.01 \\
\hline 5 & $5-5.2=-0.2$ & 0.04 & 2 & $2-2.1=-0.1$ & 0.01 \\
\hline 6 & $6-5.2=+0.8$ & 0.64 & 1 & $1-2.1=-1.1$ & 1.21 \\
\hline 6 & $6-5.2=+0.8$ & 0.64 & 3 & $3-2.1=+0.9$ & 0.81 \\
\hline 4 & $4-5.2=-1.2$ & 1.44 & 2 & $2-2.1=-0.1$ & 0.01 \\
\hline 5 & $5-5.2=-0.2$ & 0.04 & 2 & $2-2.1=-0.1$ & 0.01 \\
\hline 6 & $6-5.2=+0.8$ & 0.64 & 3 & $3-2.1=+0.9$ & 0.81 \\
\hline 4 & $4-5.2=-1.2$ & 1.44 & 2 & $2-2.1=-0.1$ & 0.01 \\
\hline 4 & $4-5.2=-1.2$ & 1.44 & 3 & $3-2.1=+0.9$ & 0.81 \\
\hline 5 & $5-5.2=-0.2$ & 0.04 & 3 & $3-2.1=+0.9$ & 0.81 \\
\hline 6 & $6-5.2=+0.8$ & 0.64 & 2 & $2-2.1=-0.1$ & 0.01 \\
\hline 5 & $5-5.2=-0.2$ & 0.04 & 3 & $3-2.1=+0.9$ & 0.81 \\
\hline 6 & $6-5.2=+0.8$ & 0.64 & 2 & $2-2.1=-0.1$ & 0.01 \\
\hline 4 & $4-5.2=-1.2$ & 1.44 & 2 & $2-2.1=-0.1$ & 0.01 \\
\hline 6 & $6-5.2=+0.8$ & 0.64 & 3 & $3-2.1=+0.9$ & 0.81 \\
\hline 5 & $5-5.2=-0.2$ & 0.04 & 1 & $1-2.1=-1.1$ & 1.21 \\
\hline 4 & $4-5.2=-1.2$ & 1.44 & 2 & $2-2.1=-0.1$ & 0.01 \\
\hline 6 & $6-5.2=+0.8$ & 0.64 & 2 & $2-2.1=-0.1$ & 0.01 \\
\hline 4 & $4-5.2=-1.2$ & 1.44 & 3 & $3-2.1=+0.9$ & 0.81 \\
\hline 5 & $5-5.2=-0.2$ & 0.04 & 3 & $3-2.1=+0.9$ & 0.81 \\
\hline 5 & $5-5.2=-0.2$ & 0.04 & 0 & $0-2.1=-2.1$ & 4.41 \\
\hline 4 & $4-5.2=-1.2$ & 1.44 & 1 & $1-2.1=-1.1$ & 1.21 \\
\hline 4 & $4-5.2=-1.2$ & 1.44 & 3 & $3-2.1=+0.9$ & 0.81 \\
\hline 6 & $6-5.2=+0.8$ & 0.64 & 2 & $2-2.1=-0.1$ & 0.01 \\
\hline \multicolumn{6}{|c|}{ Table 2. Statistics of Control Group } \\
\hline
\end{tabular}

Total $=\sum\left(\mathrm{X}_{1}-\mathrm{X}_{\mathrm{bar}}\right)^{2}=22.40$, Total $=\sum\left(\mathrm{X}_{2}-\mathrm{X}_{\mathrm{bar}}\right)^{2}=22.70$ $\mathrm{S}_{1}=\sqrt{22.40 / 30-1}=\sqrt{0.77}=0.87, \mathrm{~S} 2=\sqrt{22.70 / 29}=0.88$

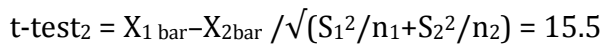

t-test $t_{1}>$ t-test ${ }_{2}=$ rejected the null hypothesis and accepted alternative hypothesis, which was significant statistically.

\begin{tabular}{|c|c|c|c|c|c|}
\hline Treatment & Headache & $\begin{array}{l}\text { Expected } \\
\text { Value (E) }\end{array}$ & $\begin{array}{c}\text { No } \\
\text { Headache }\end{array}$ & $\begin{array}{l}\text { Expected } \\
\text { Value (E) }\end{array}$ & Total \\
\hline Amitriptyline & 12 & 7 & 18 & 23 & 30 \\
\hline $\begin{array}{l}\text { Massage therapy } \\
\text { with Amitriptyline }\end{array}$ & 02 & 7 & 28 & 23 & 30 \\
\hline $\begin{array}{c}\text { Total } \\
\end{array}$ & 14 & 14 & 46 & 46 & 60 \\
\hline
\end{tabular}

$$
\begin{aligned}
& \mathrm{E} 1=\mathrm{RT} \times \mathrm{CT} / \mathrm{N}=30 \times 14 / 60=7 \\
& \mathrm{E} 2=\mathrm{RT} \times \mathrm{CT} / \mathrm{N}=30 \times 14 / 60=7 \\
& \mathrm{E} 3=\mathrm{RT} \times \mathrm{CT} / \mathrm{N}=30 \times 46 / 60=23 \\
& \mathrm{E} 4=\mathrm{RT} \times \mathrm{CT} / \mathrm{N}=30 \times 46 / 60=23 \\
& \mathrm{X}^{2}=\sum(\mathrm{O}-\mathrm{E})^{2} / \mathrm{E}=9.0 \\
& \text { Degree of Freedom }=(\mathrm{R}-1)(\mathrm{C}-1)=2-1 \times 2-1=1
\end{aligned}
$$

The null hypothesis was rejected. An alternative hypothesis was accepted. Hence massage therapy was found to be beneficial and effective.

Results are presented graphically based on the MYMOP questionnaire done by the subjects themselves.

Following is the graphic representation of each symptom, well-being and stress factors:

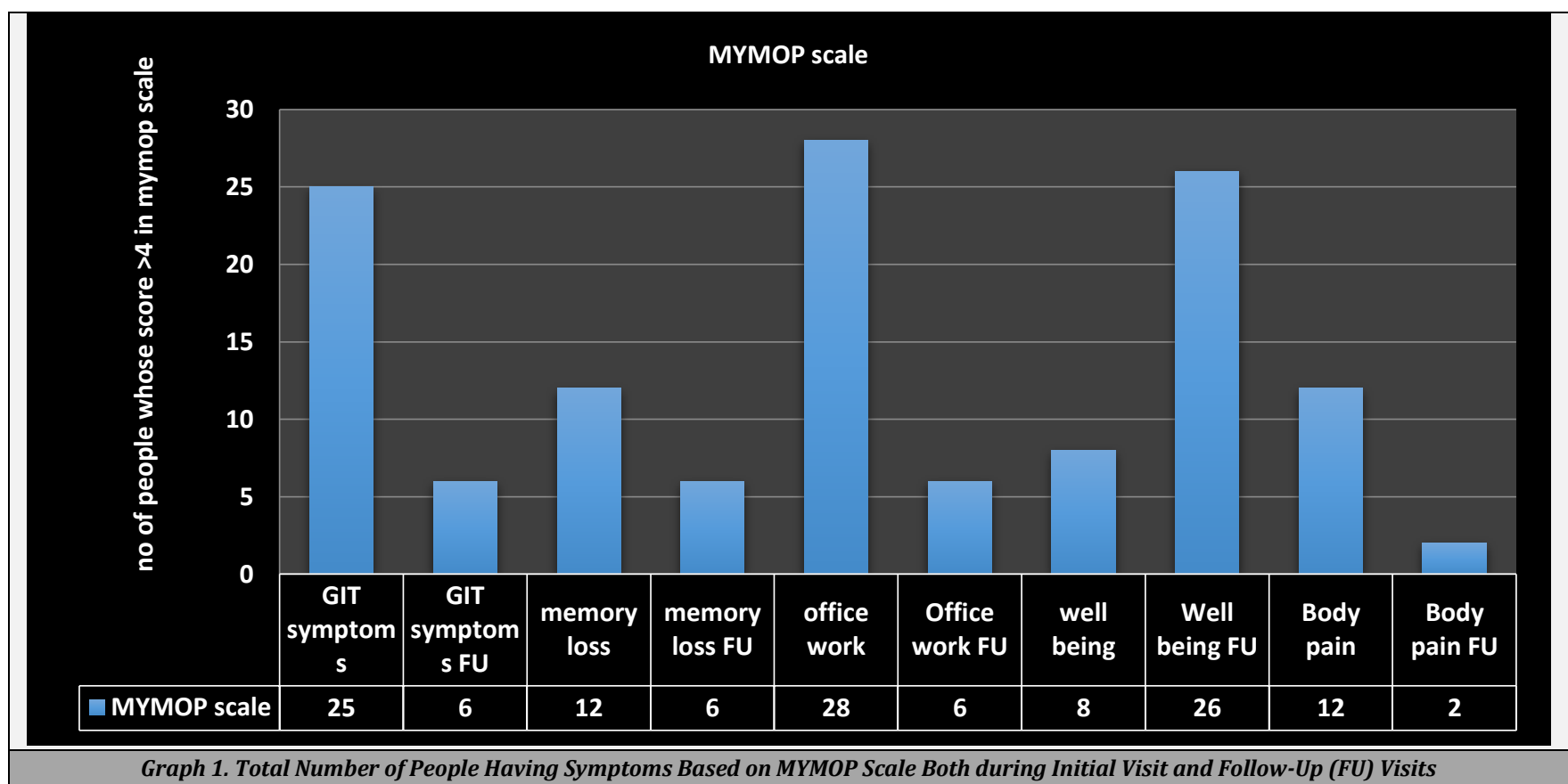

Graph 1 represents that $>80 \%$ of the subjects were having GIT symptoms (symptom 1) associated with headache. These symptoms were reduced significantly following 2 weeks of massage therapy with T. amitriptyline to $12.5 \%$.

Graph 1 represents that $>30 \%$ of the subjects were having memory loss symptoms (symptom 2) associated with headache. These symptoms were reduced significantly following 2 weeks of massage therapy with $\mathrm{T}$. amitriptyline to $15 \%$. There might be psychiatric comorbidity associated with a headache that needs to be treated separately.

Stress factors also affected significantly and stress levels came down following the massage therapy. Here we considered occupation as a stress factor. They expressed that headache disturbs the work significantly. It affects more than $90 \%$ of people during initial visits that reduced to $10 \%$ during follow up visits.

Headache affects greatly the sense of well-being. Wellbeing was found to be only $10 \%$ during the initial visit and increased to $90 \%$ during follow-up visits.

Few subjects complained of body pain associated with headache in $10 \%$. It reduced further to $2.5 \%$ during followup visits. It indicates that there is a link in body pain over headache that needs to be studied further.

Graph 2 indicates that overall, the severity of headache was found to be reduced significantly. HIT- 6 scale showed moderate to severe impact on headache scale during the initial visit, which reduced significantly to mild to no impact 
on rating scale after massage therapy in the study group compared to control group.

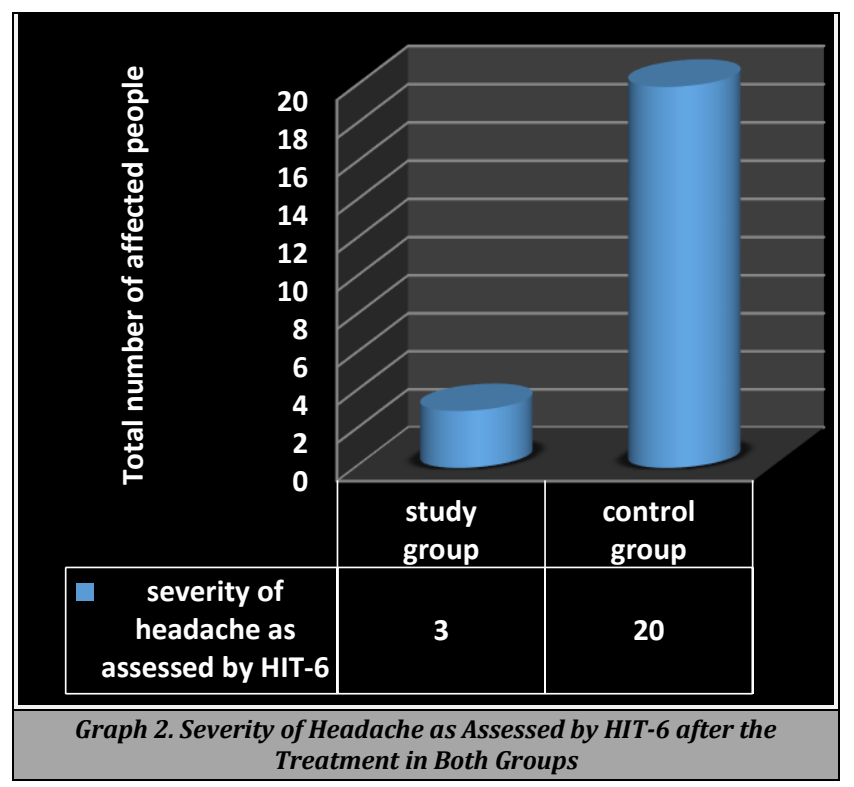

\section{DISCUSSION}

Our study had proved the effectiveness of massage therapy combined with pharmacological intervention (amitriptyline), using a self-assessed (subjective) report of the MYMOP scale and objective assessment of the HIT- 6 scale. ${ }^{18}$ MYMOP scales ${ }^{19}$ benefited in assessing 3 associated symptoms along with headache which are GIT symptoms, memory loss and body pains.

Massage therapy has shown to be effective in treating headaches along with associated symptoms. Our study had proved that headache is reduced significantly following two weeks of massage therapy combined with pharmacological intervention (amitriptyline).

HIT- 6 scale is an objective scale that helps to assess the severity of headache which reduced significantly to nil-mild intensity following two weeks of massage therapy. Since both act at different sites, receptor sites and trigger points, our study is effective in reducing headaches along with associated symptoms. Also, MYMOP \& HIT-6 score changes significantly following the massage regimen. Even though the associated symptoms like GIT symptoms, memory loss, stress factors and body pain reduced significantly the mechanism of improvement in them is not known, yet to be proved. A decline in severity of headache has been maintained throughout the massage regimen.

Some researchers believed that touching pressure points in a certain way can: ease pain, whether they cause, or are a manifestation of, the headache, however, is not clear, since not every patient with a headache has active TrPs. There is evidence to suggest that $\mathrm{TrPs}$ may initiate a peripheral nociceptive mechanism that contributes to changes in the central nervous system (CNS). Sensitization of nociceptive pain pathways secondary to persistent nociceptive activity from active TrPs may then contribute to the progression from acute to more chronic headache attacks. According to the classic TrP doctrine described by Simons and Travell, an active $\operatorname{TrP}$ has the following characteristics: is tender and is located in a firm or taut band of the muscle refers to pain on deep palpation displays a "twitch" response under the palpating fingers.

In one of the researches, the authors noted a decrease in neck pain after the subjects received 10 one-hour upper body massages over 2 weeks. Although the treatment was effective, the massage regimen employed was not realistic for most patients; furthermore, the therapeutic massage procedures were not clearly described. Their study also proved that the massage administered during a headache might result in immediate beneficial effects.

Our study proved that headache associated with other symptoms like GIT symptoms, memory loss among the study group had been alleviated effectively through the combined therapy among study as compared to control group. It indicates that there is a link between associated symptoms with a headache that needs to be studied further. Since both act at different sites viz massage acts on MTrPs and drugs act at the receptor level. Although there are various diagnostic tools available like ultrasonography, microdialysis, EMG, MRI and Infrared thermography, they are not gold standard diagnostic tools to assess the MTrPs.

\section{CONCLUSIONS}

Our study had proved that combined therapy is very effective as compared to the control group and was statistically significant. T. amitriptyline acts only at the receptor level and hence headache is not resolved completely in most of the patients. Since both massage therapy and medications act at different sites, our study proved that combined therapy is effective in reducing headaches along with associated symptoms significantly.

\section{Limitations}

Our study had a small sample size due to the difficulty to convince the subject for massage therapy. Most of them were not willing to continue the massage regimen further to see the long-term consequences.

Data sharing statement provided by the authors is available with the full text of this article at jemds.com.

Financial or other competing interests: None.

Disclosure forms provided by the authors are available with the full text of this article at jemds.com.

\section{REFERENCES}

[1] Saylor D, Steiner TJ. The Global burden of headache. Semin Neurol 2018;38(2):182-90.

[2] Quinn C, Chandler C, Moraska A. Massage therapy and frequency of chronic tension headaches. Am J Public Health 2002;92(10):1657-61.

[3] https://www.betterhealth.vic.gov.au/health/Conditions AndTreatments/massage 
[4] Myers DE, McCall WD Jr. Head pain as a result of experimental ischemic exercise of the temporalis muscle. Headache 1983;23(3):113-6.

[5] Simons DG, Travell JG, Simons LS. Travell \& Simons' Myofascial pain and dysfunction: the trigger point manual. $2^{\text {nd }}$ edn. Baltimore, Md: Williams \& Wilkins 1999.

[6] Mennell J. Myofascial trigger points as a cause of headaches. J Manipulative Physiol Ther 1989;12(4):30813.

[7] Lipton RB, Bigal ME, Steiner TJ, et al. Classification of primary headaches. Neurology 2004;63(3):427-35.

[8] Nicholson RA, Houle TT, Rhudy JL, et al. Psychological risk factors in headache. Headache 2007;47(3):413-26.

[9] Goadsby PJ, Raskin NH. Headache. Chap - 14. In: Longo DL, Fauci AS, Kasper DL, et al. eds. Harrison's Principles of Internal Medicine. $18^{\text {th }}$ edn. New York, NY: McGrawHill 2012.

[10] Greenberg D, Aminoff M, Simon R. Clinical Neurology. Chap - 6. Headache \& facial pain. $8^{\text {th }}$ edn. McGraw-Hill Professional 2012.

[11] Edlow JA, Panagos PD, Godwin SA, et al. Clinical policy: critical issues in the evaluation and management of adult patients presenting to the emergency department with acute headache. Annals of Emergency Medicine 2008;52(4):407-36.
[12] Airaksinen O, Pontinen PJ. Effects of the electrical stimulation of myofascial trigger points with tension headache. Acupunct Electrother Res 1992;17(4):285-90.

[13] Rubin D. Myofascial trigger point syndromes: an approach to management. Arch Phys Med Rehabil 1981;62(3):107-10.

[14] Hubbard DR, Berkoff GM. Myofascial trigger points show spontaneous needle EMG activity. Spine 1993;18(13):1803-7.

[15] Davidoff RA. Trigger points and myofascial pain: toward understanding how they affect headaches. Cephalalgia 1998;18(7):436-48.

[16] Schwartz BS, Stewart WF, Lipton RB. Lost workdays and decreased work effectiveness associated with headache in the workplace. J Occup Environ Med 1997;39(4):3207.

[17] Headache Classification SubCommittee of The International Headache Society. The International Classification of Headache Disorders: 2nd edn. Cephalalgia 2004;24(Suppl 1):9-160.

[18] Kosinski M, Bayliss MS, Bjorner JB, et al. A six-item shortform survey for measuring headache impact: the HIT-6. Qual Life Res 2003;12(8):963-74.

[19] Paterson C, Britten N. In pursuit of patient-centred outcomes: a qualitative evaluation of the 'Measure Yourself Medical Outcome Profile'. J Health Serv Res Policy 2000;5(1):27-36. 